\title{
Research on an Improved Ant Colony Optimization Algorithm for Solving Traveling Salesmen Problem
}

\author{
Wenli Lei ${ }^{1,2^{*}}$ and Fubao Wang ${ }^{1}$ \\ ${ }^{1}$ School of Electronics and Information, Northwestern Polytechnical University, \\ Xi'an, 710072 China \\ ${ }^{2}$ School of Physics and Electronic Information, Yan'an University, Yan'an 716000
} China

\begin{abstract}
In order to improve the search result and low evolution speed, and avoid the tendency towards stagnation and falling into the local optimum of ant colony optimization(ACO) in solving the complex function, the traditional ant colony optimization algorithm is analyzed in detail, an improved ant colony optimization(IWSMACO) algorithm based on information weight factor and supervisory mechanism is proposed in this paper. In the proposed IWSMACO algorithm, the information weight factor is added to the path selection and pheromone adjustment mechanisms in order to dynamically adjust path selection probability and randomly select the behavior rules for further intelligentializing the ant colony. The supervisory mechanism added the dynamic convergence criterion of supervisory distance and used the optimal pheromone update strategy to self-adaptively select the excellent ants for updating the pheromone trails, and improve the solution qualities of each iteration, better guide the later ants for learning. Finally, the proposed IWSMACO algorithm is carried out by 12 TSP instances. The simulation experiment results show that the proposed IWSMACO algorithm can not only avoid falling into the local optimum, but also enhance the convergence speed. And it takes on remarkable optimized ability and higher search accuracy.
\end{abstract}

Keywords: ant colony optimization algorithm, information weight factor, supervisory mechanism, convergence criterion, function optimization

\section{Introduction}

Ant colony optimization(ACO) algorithm is a bio-inspired optimization algorithm, which simulates the swarm intelligence behavior of ants. It was originally proposed by Dorigo at the beginning of 1990s [1]. The ACO algorithm simulates the foraging process of ants. The ants release the pheromone trails on the passed path in order to record the passed path in the searching food. The other ants will select the path according to the perception of pheromone concentration. The pheromone concentration can enhance or decay. When one path passes more ants, the pheromone concentration will be increase on this passed path, and more ants are attracted to choose this path. If the path passes less ants, the pheromone concentration gradually reduced to lead to choose this path with small probability. Ultimately, most ants are focused on the shortest path from the source. The ACO algorithm has strong robustness, distributed cooperation and so on, it has been widely applied in traveling salesman path optimization, shop scheduling, logistics distribution, data mining, image processing, network communication, and so on[2-6]. The ACO algorithm become one of the most effective algorithms to solve combination optimization problem.

The ACO algorithm is a swarm intelligence algorithm based on priori information and the positive feedback mechanism of pheromone. The core idea is to guide the behavior of each ant by using the pheromone concentration in order to gradually move towards the 
global shortest path for the whole ant colony. So the information processing method has a great influence on the efficiency and accuracy of the whole algorithm. But because the ACO algorithm uses the random probability selection and the pheromone positive feedback strategy, it has many problems, such as poor stability, slow convergence speed, stagnation phenomenon, easy to fall into local optimal solution and so on. In order to better improve these existing problems, many researchers proposed a lot of improved ACO algorithms. Janson et al. [7] studied the problem of executing ant colony optimization(ACO) algorithms on a dynamically reconfigurable mesh architecture. Mitica and Catalin [8] proposed an improved decentralized Load Balance (LB) paradigm based on ACO metaheuristic for P2P Networks. The experiments were done with both constant and variable heuristic value and comparisons were made. Leng et al. [9] proposed an improved ant colony optimization (ACO) algorithm based on the initial weighted directional diagram and the dynamic pheromone updating in cell scheduling of the flexible manufacturing process for considering the instrument constraint, manufacturing cost and time. Duan et al. [10] proposed an application of ant colony optimization algorithm based on Grid to tune the parameters in the design of a novel type of nonlinear proportional-integral-differential(NLPID) controller, which is used in flight simulator. Abadeh et al. [11] proposed an evolutionary algorithm to induct fuzzy classification rules. The algorithm uses an ant colony optimization based local searcher to improve the quality of final fuzzy classification system. Huang [12] proposed a novel hybrid ACO-based classifier model that combines ant colony optimization(ACO) and support vector machines(SVM) to improve classification accuracy with a small and appropriate feature subset. The experimental results indicate that the hybridized approach can correctly select the discriminating input features and also achieve high classification accuracy. Li and Tian [13] proposed an improved ant colony algorithm for the search of the optimal path and the establishment of the multicast tree to meet QoS constraints in multicast routing. Experiments show that the algorithm has a better performance than the other routing algorithm and establish the multicast tree quickly. Geng et al. [14] proposed an improved solution algorithm using ant colony optimization (ACO) for finding global optimum for any given test functions. The proposed algorithm is based on each ant searches only around the best solution of the previous iteration with $\beta$. Zhang and Tang [15] proposed a novel hybrid ant colony optimization approach called SS_ACO algorithm to solve the vehicle routing problem. The main feature of the hybrid algorithm is to hybridize the solution construction mechanism of the ant colony optimization(ACO) with scatter search (SS).Yi and Lai [16] proposed a new ant colony algorithm to deal with $\mathrm{p} / / \mathrm{T}(\mathrm{p} / / \mathrm{T}-\mathrm{ACO})$, and designed he computing model of mapping $\mathrm{p} / / \mathrm{T}$ to the ant colony optimization environment. The theoretical analysis and comparative experiments demonstrate that $\mathrm{p} / / \mathrm{T}-\mathrm{ACO}$ has much better performance and can be used to solve practical large scale problems efficiently. Jiang et al.[17] proposed a dynamic ant colony optimization (ACO) algorithm to solve dynamic traffic routing problem. The main objective of this work is to search out the least-time-cost route in a variable-edge-weight graph. Shuang et al. [18] proposed a hybrid PS-ACO algorithm based on ACO algorithm and particle swarm optimization (PSO) algorithm. The pheromone updating rules of ACO are combined with the local and global search mechanisms of PSO. The convergence analysis and parameters selection are given through simulations on traveling salesman problems (TSP). Janaki Meena et al. [19] proposed an ant colony optimization(ACO) algorithm to select features for categorizing longer documents to closely related categories. Experiments were conducted with documents from 20newsgroup and Reuters-21578 benchmarks. Geng et al. [20] proposed a directional ant colony optimization(DACO) algorithm for solving nonlinear resource-leveling problems. The DACO algorithm introduced can efficiently improve the convergence rate and the quality of solution for real-project scheduling. Xing et al. [21] proposed a hybrid ant colony optimization(ACO) algorithm(HACOA) to solve instances of the extended capacitated arc routing problem(CARP). This approach is 
characterized by the exploitation of heuristic information, adaptive parameters, and local optimization techniques. Cao et al. [22] proposed a novel ant colony optimization algorithm to establish minutiae correspondences in large-distorted fingerprints. The proposed method is tested on FVC2004 DB1 and a FINGERPASS cross-matching database established by our lab. Li et al. [23] proposed a promising modification with changing index in order to solve the premature convergence problem of the basic ant colony optimization algorithm and find an effective method for the calculation of system reliability index of the structure. Zhao et al. [24] proposed a hybrid ant colony optimization algorithm by introducing extremal optimization local-search algorithm to the ant colony optimization(ACO) algorithm, and is applied to multiuser detection in direct sequence ultra wideband (DS-UWB) communication system. Janaki Meena et al. [25] proposed the text feature selection problem as a combinatorial problem and an ant colony optimization algorithm to find the nearly optimal solution for the same. Chen et al. [26] proposed a fast two-stage ACO algorithm based on the scent pervasion principle for overcoming the inherent problems of traditional ACO algorithms. The basic idea is to split the heuristic search into two stages: preprocess stage and path planning stage. Hsu and Juang [27] proposed an evolutionary wall-following control of a mobile robot using an interval type-2 fuzzy controller(IT2FC) with species-differential-evolution-activated continuous ant colony optimization(SDE-CACO). The proposed SDE-CACO is compared with various population-based optimization algorithms to demonstrate its efficiency and effectiveness in the wall-following control problem. Li et al. [28] proposed an improved Ant Colony Optimization(ACO) algorithm to solve the subjectivity problem of resource scheduling in construction project group management. Ke et al. [29] proposed a multiobjective EA, i.e., MOEA/D-ACO based on combining ant colony optimizatio(ACO) and the multiobjective evolutionary algorithm (EA). We also demonstrate that the heuristic information matrices in MOEA/D-ACO are crucial to the good performance of MOEA/D-ACO for the knapsack problem. Elloumi et al. [30] proposed a new hybrid method (PSO-ACO) based on Particle swarm optimization (PSO) and Ant colony optimization (ACO) algorithms for solving the traveling salesman problem (TSP). Shima and Hossein [31] proposed a novel feature selection algorithm based on ant colony optimization, called Advanced Binary ACO(ABACO). The performance of proposed algorithm is compared to the performance. Zhang and Tang [32] proposed a hybrid configuration of ant colony optimization( $\mathrm{ACO}$ ) with artificial bee colony (ABC) algorithm, called hybrid ACO-ABC algorithm for optimal location and sizing of distributed energy resources(DERs) (i.e., gas turbine, fuel cell, and wind energy) on distribution systems. Xiong et al. [33] proposed an improved multi-objective ant colony optimization algorithm considering the timeliness of goal nodes to generate the node sequence.

Although the improved ACO algorithm takes on better optimization performance for solving complex problems, but the low evolution speed and stagnation still exists in these improved ACO algorithm. So the information weight factor and supervisory mechanism are introduced into the basic ACO algorithm in order to improve the low evolution speed and avoid the tendency towards stagnation. An improved ant colony optimization (IWSMACO) algorithm is proposed to improve the low evolution speed and the solution qualities of each iteration, and avoid the tendency towards stagnation.

The rest of this paper is organized as follows. Section 2 briefly introduces the traveling salesmen problem. Section 3 briefly introduces the ant colony optimization(ACO) algorithm. Section 4 briefly introduces the improved ACO algorithm based on information weight factor and supervisory mechanism. Section 5 gives TSP numerical experiment and results analysis. Finally, the conclusions are discussed in Section 6. 


\section{Traveling Salesmen Problem}

Traveling salesmen problem(TSP) [34] is has specifically commanded much attention of mathematicians and computer scientists, because it is easy to describe and difficult to solve. The TSP can be represented by using a complete directed graph $G=(N, A)$, where $N$ is a set of $n$ nodes (vertices), also called cities, and $A$ is a set of arcs and $D=d_{i j}$ is the associated distance matrix with each $\operatorname{arc}(i, j) \in A$. The distance matrix $D$ can be either symmetric or asymmetric. The TSP is to find one shortest closed tour visiting each one of the $n=|N|_{\text {nodes of graph }} G$. The distance between the cities are independent of the direction of traversing the arcs, that is, $d_{i j}=d_{j i}$ for every pair of nodes in symmetric TSP. In the asymmetric TSP, there exists the $d_{i j} \neq d_{j i}$ for at least one pair of nodes $(i, j)$. All TSP instances from the TSPLIB benchmark library are used in this paper.

Define the variables:

$$
x_{i j}= \begin{cases}1 & \text { if the } \operatorname{arc}(i, j) \text { is in the tour } \\ 0 & \text { otherwise }\end{cases}
$$

The TSP can be formulated by the following generalization with a well known integer program formulation.

Objective function:

$$
z=\min \sum_{i} \sum_{j} d_{i j} x_{i j}
$$

The constraints are described as follows:

$$
\begin{aligned}
& \sum_{i=1}^{n} x_{i j}=1, j=1,2,3, \cdots, n \\
& \sum_{j=1}^{n} x_{i j}=1, i=1,2,3, \cdots, n \\
& x_{i j} \in\{0,1\}, i, j=1,2,3, \cdots, n \\
& \sum_{i, j \in S}^{n} x_{i j} \leq|S|-1,2 \leq|S| \leq N-2
\end{aligned}
$$

In these formulations, the objective function (2) describes the total cost for being minimized. Constraint(3) ensures that each position $j$ is occupied by only one city, while constraint(4) guarantees that each city $i$ is assigned to the exact position. Constraint (5) describes the integrality constraints of variables zero-one $x_{i j}\left(x_{i j} \geq 0\right)$. Constraint(6) assures that each city in the final route will be visited once.

\section{Ant Colony Optimization(ACO) Algorithm}

The ACO algorithm consists of a number of cycles of solution construction. During each iteration, a number of ants construct complete solutions by using heuristic information and the collected experiences of previous population of ants. These collected experiences are represented by the pheromone trail. The less quantities are deposited during the construction phase, while larger amounts are deposited at the end of each iteration. Pheromone can be deposited on the components and/or the connections in one solution. Each ant randomly starts at one city and visits the other cities according to the 
transition rule. After the ants complete their routes, the system will evaluate the length of the routes. And the system uses the pheromone update rule to update the pheromone information. The learning procedure is to update the pheromone information repeatedly. The procedure of the ACO algorithm is given in Figure 1.

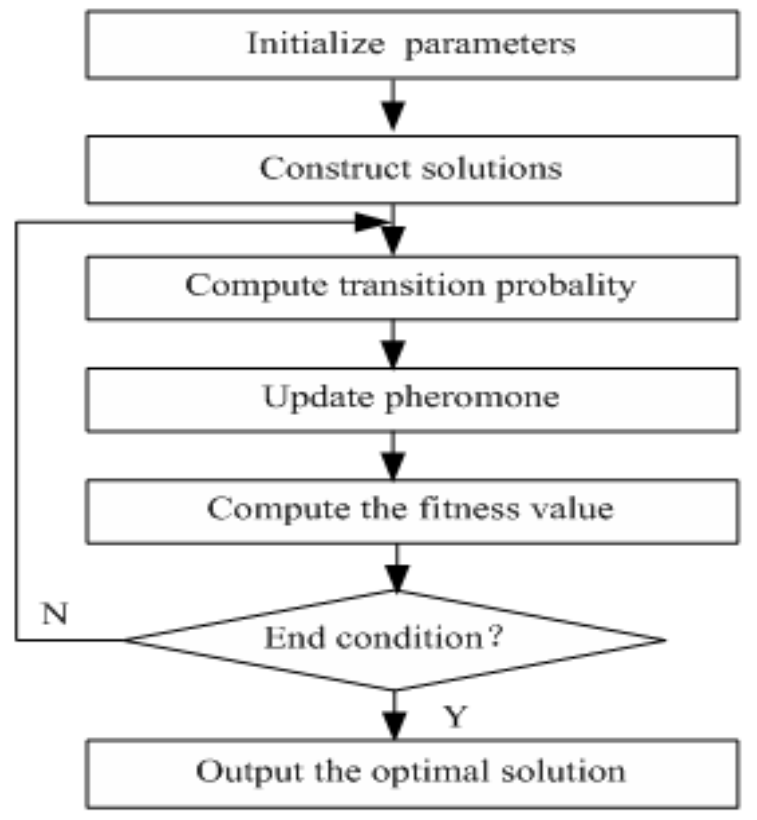

Figure 1. The Flow of the ACO Algorithm

(1) Transition rule

In the route, the $k^{\text {th }}$ ant starts from city ${ }^{i}$, the next city $j$ is selected among the unvisited cities memorized in $J_{r}^{k}$ according to the following formula:

$$
s=\underset{u \in J_{r}^{k}}{\arg \max }\left[\tau_{i}(t)^{\alpha} \cdot \eta(t)^{\beta}\right] \text { if } q \leq q_{0}(\text { Exploitation })
$$

To visit the next city s with the probability $p_{k}(r, s)$,

$$
p_{i j}(t)= \begin{cases}\frac{\tau_{i j}(t)^{\alpha} \cdot \eta_{i j}(t)^{\beta}}{\sum_{u \in J_{r}^{k}} \tau_{i s}(t)^{\alpha} \cdot \eta_{i s}(t)^{\beta}} & \text { if } s \in J_{r}^{k} \\ 0 & \text { otherwise } \\ 0 & \text { if } q>q_{0}(\text { Bias Exploitation })\end{cases}
$$

In two formula, $p_{i j}(t)$ is the transition probability, $\tau_{i j}(t)$ is the intensity of pheromone between the city $i$ andt he city $j, \eta_{i j}(t)$ is the length of the path from the city $i$ to the city $j, J_{r}^{k}$ is the set of unvisited cities of the $k^{\text {th }}$ ant, the parameter $\alpha$ and $\beta$ are the control parameters, $q$ is a uniform probability $[0,1]$.

(2) The pheromone update rule

In order to improve the solution, the pheromone trails must be updated. Trail updating includes local updating and global updating. The local trail updating formula is given by:

$$
\tau_{i j}(t)=(1-\rho) \tau_{i j}(t)+\sum_{k=1}^{m} \Delta \tau_{k}(t)
$$

In the formula (9), $\rho(0<\rho<1)$ is the pheromone trial evaporating rate. $\Delta \tau_{k}(t)$ is 
the amount of pheromone trail added to the edge $\left({ }^{i, j}\right)$ by ant $k$ between time ${ }^{t}$ and $t+\Delta t$ in the tour. It is given by:

$$
\Delta \tau_{k}(t)= \begin{cases}\frac{Q}{L_{k}} & (i, \mathrm{j}) \in \pi_{k} \\ 0 & \text { otherwise }\end{cases}
$$

Where $Q$ is a constant parameter, ${ }^{L_{k}}$ is the distance of the sequence ${ }^{\pi_{k}}$ toured by ant in $\Delta t$.

\section{Improved ACO Algorithm Based on Information Weight Factor and Supervisory Mechanism}

\subsection{The Information Weight Factor}

The ACO algorithm is a kind of positive feedback heuristic algorithm, it is to obtain the better solution according to the left information of the ants on each path. If the amount of information is greater, the chance of selection is greater. But when the evolution is a certain iteration, the number of ants will be gathered in a few paths due to the increasing amount of information. This will result in the premature and stagnation and obtain the local optimal solution. So the path selection probability and pheromone updating mechanism are dynamically adjusted according to the solution distribution in the optimization process of ACO algorithm, in order to avoid the premature and stagnation and improve the convergence speed and stability of ACO algorithm.

In order to avoid to fall into the local optimal solution, it is necessary to adjust the selection probability of each path. The information weight factor is used to limit the information and expectation degree for the selection probability of ant. The $r$ paths from the ${ }^{i}$ city are sorted from the high to the low according the information amount. The sorted result is stored in the array rank, the value of the array element $\operatorname{rank}[i]$ is the serial number of path $(i, j)$.

$$
\xi_{i j}= \begin{cases}\left(\frac{\omega}{r}\right)^{\operatorname{rank}[j]-1} & , \text { if } \operatorname{rank}[j] \leq \omega \\ 0 & , \text { other }\end{cases}
$$

Where $\omega$ is the selecting path of the ${ }^{i}$ city, $\xi_{i j}$ is application information weight of path $(i, j)$. The probability of selecting the ${ }^{j}$ city from the ${ }^{i}$ city is described as follow:

$$
p_{k}(i, j)= \begin{cases}\frac{\xi_{i j} \times \tau(i, j)^{\alpha} \times \eta\left(i, j^{\beta}\right.}{\sum_{u \in J_{r}^{k}}^{k} \xi_{i u} \tau(i, s)^{\alpha} \cdot \eta(i, s)^{\beta}} & \text { if } s \in J_{r}^{k} \\ 0 & \text { otherwise } \\ & \text { if } q>q_{0} \text { (Bias Exploitation) }\end{cases}
$$

When the ant travels the path of the starting from the ${ }^{i}$ city that is concentrated in the last iteration, the aggregation degree of the ${ }^{i}$ city is larger, and the $\omega$ of selected path is also larger. The information weight $\xi_{i j}$ of each path is relatively close. The next iteration, the selected probability of each path is more uniform. When the ant travels the path of the starting from the ${ }^{i}$ city that is scattered in the last iteration, the aggregation degree of the 


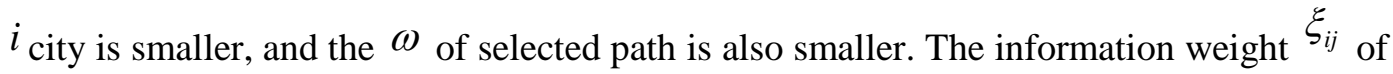
each path is relatively larger.

\subsection{The Supervisory Mechanism}

In the engineering construction, a project supervision is sent to be responsible for supervision construction quality. When the quality achieves the standard, the next task is allowed to perform by workers. Otherwise, the workers are required to rework. This mechanism ensures the construction quality. A virtual adaptive criterion -- taskmaster distance is used to evaluate the passed path distance of ant in this paper.

$$
L_{j}(t+1)=\frac{\min \left(L_{\text {avg }}(1: t)\right) \times w+L_{\text {best }}(t)+L_{j}(t)}{w+2}
$$

where $\min \left(L_{\text {avg }}(1: t)\right)$ is the shortest average distance from the first iteration to the $t^{\text {th }}$ iteration, $L_{b e s t}(t)$ is the global minimum distance of the $t^{t h}$ iteration, $w$ is a constant. From the relationship of mathematics, $L_{j}(t+1)$ is monotonically decreasing, which means supervision standard gradually improve in order to ensure the convergence of the algorithm.

If the ant passes the path distance is longer than the taskmaster distance, then the ant is required to rework until less than the taskmaster distance. Of course, in order to prevent the larger calculation, the reworked number is set an upper limit $b$. When the ant passes the whole path, the path of less than taskmaster distance is globally updated and adaptively selects the number of ants with higher quality. By adding the taskmaster distance, the solution quality of each iteration is improved, and can guide the ant to better learn in order to obtain the better solution.

It is known from the formula(13), when the algorithm falls into the local optimum, with the increasing of iteration $t$, the $L_{j}(t+1)$ is gradually close to the $L_{\text {best }}(t+1)$ or equal to the $L_{b e s t}(t+1)$. At this time, all the paths are only locally updated, and the information is increased. The path with more pheromone is selected with the high probability, he pheromone concentration rapidly decreases to increase the opportunity for choosing the other path, which is helpful to jump out the local optimum. At the same time, in order to prevent the low pheromone concentration of the global optimal path, in the first iteration, if there is no one ant to choose the global optimal path, the path is globally updated according the formula(14) in order to ensure that the ant will not deviate from the global optimal path. This method also has the adaptive function. When the $\tau(i, j)$ is relatively small, it will increase the more.

$$
\begin{aligned}
& \quad \tau_{i j}^{m}(t) \leftarrow(1-\lambda) \times \tau_{i j}^{m}(t)+\left[\tau_{\text {best }}(t)-\tau_{i j}(t)\right] / \tau_{\text {best }}(t) \times n_{\text {best }} \times \lambda \times Q \times L_{\text {avg }} / L^{2} \\
& \text { where } \tau_{\text {best }}(t)=\max \left(\tau_{i 1}(t), \tau_{i 2}(t), \cdots, \tau_{\text {in }}(t)\right),{ }^{n_{\text {best }}}>1 .
\end{aligned}
$$

\section{TSP Numerical Experiment and Results Analysis}

In order to analyze and prove the solving ability of the improved ACO(IWSMACO) algorithm, 12 TSP datasets are used from the TSPLIB standard library. The scale of cities in these TSP datasets are from 48 to 14051. And the basic ACO algorithm and improved ACO(QACO) algorithm based on quantum evolutionary algorithm are selected to compare the optimized performance with the proposed IWSMACO algorithm. The distance of each two cities is computed by using Euclidian distance. It is a very 
complicated problem to obtain the parameters' values of the algorithm, because the changes of parameters' values could seriously affect solving the optimum value. So the most reasonable initial parameters' values are obtained by testing and modifying. The obtained initial parameters' values are: ants $m=50$, pheromone amount $Q=80$, pheromone factor $\alpha=1.0$, heuristic factor $\beta=2.0$, initial concentration $\tau_{i j}(0)=1.5$, evaporation factor $\rho=0.15$, maximum iteration times $T_{\max }=500$, $n_{\text {best }}=8, b=5,{ }^{m=\frac{n}{2} \sim n}$, initial concentration $\tau_{i j}(0)=1 /\left(L_{g} * m\right), L_{g}$ is the estimated shortest distance. The experiment environments are: the Pentium CPU 2.40GHz, 4.0GB RAM with Windows XP and Matlab2012. For each TSP, the basic ACO algorithm, improved $\mathrm{ACO}(\mathrm{QACO})$ algorithm and IWSMACO algorithm are independently run 20 times. In this paper, the best value and average value are selected to described the optimization performance of these algorithms. The numerical experiment results are shown in Table 1.

Table 1. The Numerical Experiment Results for Selected TSP

\begin{tabular}{|c|c|c|c|c|c|c|c|}
\hline \multirow{2}{*}{ Instances } & \multirow{2}{*}{$\begin{array}{c}\text { Optimal } \\
\text { Value }\end{array}$} & \multicolumn{2}{|c|}{ Basic ACO algorithm } & \multicolumn{2}{|c|}{ QACO algorithm } & \multicolumn{2}{|c|}{ IWSMACO algorithm } \\
\hline & & Best value & Avg. value & Best value & Avg. value & Best value & Avg. value \\
\hline att 48 & 33522 & 34078 & 343562 & 33804 & 34158 & 33524 & 33737 \\
\hline eil51 & 426 & 445 & 466 & 433 & 449 & 426 & 441 \\
\hline st70 & 675 & 696 & 721 & 686 & 710 & 680 & 704 \\
\hline eil76 & 538 & 578 & 591 & 551 & 565 & 546 & 559 \\
\hline kroD100 & 21294 & 21964 & 23392 & 22006 & 22406 & 21394 & 22319 \\
\hline $\operatorname{ch} 130$ & 6110 & 6156 & 6191 & 6129 & 6179 & 6118 & 6145 \\
\hline rat195 & 2323 & 2407 & 2562 & 2395 & 2489 & 2346 & 2451 \\
\hline rd400 & 15281 & 15453 & 15546 & 15358 & 15482 & 15356 & 15448 \\
\hline rat783 & 8806 & 9146 & 9403 & 9102 & 9315 & 9037 & 9210 \\
\hline $\mathrm{d} 1291$ & 50801 & 53137 & 54462 & 52953 & 53970 & 52385 & 53146 \\
\hline nl4461 & 182566 & 19283 & 20134 & 19103 & 19651 & 18935 & 19204 \\
\hline brd 14051 & 469385 & 477301 & 479069 & 476621 & 476898 & 476352 & 476767 \\
\hline
\end{tabular}

As can be seen from Table 1, the proposed IWSMACO algorithm can obtain better optimal value than the basic ACO algorithm and QACO algorithm for all 12 TSP instances on the best value and average value. And att48, st70, eil76 and ch130 find the best value that is close to the known value by using proposed IWSMACO algorithm. The proposed IWSMACO algorithm can find the best known value $\mathbf{4 2 6}$ for the eil51instance. For larger scale instance, the numerical experiment results that the proposed IWSMACO algorithm can obtain better optimization value. So the experiment shows that the IWSMACO algorithm is more capable to solve the complex optimization problem, can 
improve the search result and low evolution speed and avoid the tendency towards stagnation and falling into the local optimum. The proposed IWSMACO algorithm takes on the better optimization performance than the basic ACO algorithm and QACO algorithm.

\section{Conclusion}

The ACO algorithm is a bio-inspired optimization algorithm by simulating the swarm intelligence behavior of ants. It has strong robustness, distributed cooperation and so on. But in the application, it exists premature convergence, poor search ability and easy falling into local optimal solutions in solving complex optimization problem. So the information weight factor and supervisory mechanism are introduced into the basic ACO algorithm in order to improve the low evolution speed and avoid the tendency towards stagnation. The information weight factor is used to dynamically adjust path selection probability and randomly select the behavior rules for further intelligentializing the ant colony. The supervisory mechanism used the dynamic convergence criterion of supervisory distance and the optimal pheromone update strategy to self-adaptively select the excellent ants for updating the pheromone trails, and improve the solution qualities of each iteration. So an improved ant colony optimization (IWSMACO) algorithm is proposed. And 12 TSP datasets are used from the TSPLIB standard library in order to analyze and prove the solving ability of the improved ACO(IWSMACO) algorithm. The experiment shows that the IWSMACO algorithm is more capable to solve the complex optimization problem, can improve the search result and low evolution speed and avoid the tendency towards stagnation and falling into the local optimum.

\section{Acknowledgments}

The authors would like to thank all the reviewers for their constructive comments. The authors acknowledge with thanks the Scientific Research Program Funded by Shaanxi Provincial Education Department (Program No. 14JK1829), Yan'an Municipal Science and Technology Research and Development Program(Program No. 2014KG-04), the Science and Technology Research and Development Program Project in Shaanxi Province (Industrial Research) (Grant No.2014K05-61), and the National Nature Science Foundation of China (Grant No. 61379026). The program for the initialization, training, and simulation of the proposed algorithm in this article was written with the tool-box of MATLAB 2010 produced by the Math-Works, Inc.

\section{References}

[1] M. Dorigo and M. Gambardellal, "Ant colony system: A cooperative learning approach to the traveling salesman problem", IEEE Transactions on Evolutionary Computation, vol.1, no. 1, (1997), pp. 53-66.

[2] W. Deng, R. Chen, B. He, Y. Q. Liu, L. F. Yin and J. H. Guo, "A novel two-stage hybrid swarm intelligence optimization algorithm and application", Soft Computing, vol. 16, no. 10, (2012), pp. 17071722 .

[3] M. R. Jalali, A. Afshar and M. A. Mariño, "Improved ant colony optimization algorithm for reservoir operation", Scientia Iranica, vol. 13, no. 3, (2006), pp. 295-302.

[4] W. Deng, H. M. Zhao, J. J. Liu, X. L. Yan, Y. Y. Li, L. F. Yin and C. H. Ding, "An improved CACO algorithm based on adaptive method and multi-variant strategies", Soft Computing, vol. 19, no. 3, (2015), pp. 701-713.

[5] Q. X. Zheng, M. Li, Y. X. Li and Q. H. Tang, "An improved ant colony optimization for two-sided assembly line balancing problem", Acta Electronica Sinica, vol. 42, no. 5, (2014), pp. 841-845

[6] Y. G. Zhang, S. B. Zhang and Q. S. Xue, "Improved ant colony optimization algorithm for solving constraint satisfaction problem", Journal on Communications, vol. 36, no. 5, (2015), pp. 1-6.

[7] S. Janson, D. Merkle, M. Middendorf, H. Elgindy and H. Schmeck, "On Enforced Convergence of ACO and its Implementation on the Reconfigurable Mesh Architecture Using Size Reduction Tasks", Journal of Supercomputing, vol. 26, no. 3, (2003), pp. 221-238.

[8] C. Mitica and B. Catalin, "An improved load balance strategy using ACO metaheuristics ", WSEAS Transactions on Computers, vol. 4, no. 8, (2005), pp. 960-965. 
[9] S. Leng, X. B. Wei, W. Y. Zhang, "Improved ACO scheduling algorithm based on flexible process", Transactions of Nanjing University of Aeronautics and Astronautics, vol. 23, no. 2, (2006), pp. 154-160.

[10] H. B. Duan, D. B. Duan and X. F. Yu, "Grid-based ACO algorithm for parameters tuning of NLPID controller and its application in flight simulator", International Journal of Computational Methods, vol. 3, no. 2, (2006), pp. 163-175.

[11] M. S. Abadeh, J. Habibi and E. Soroush, "Induction of fuzzy classification systems via evolutionary ACO-based algorithms", International Journal of Simulation: Systems, vol. 9, no. 3, (2008), pp. 1-8.

[12] C. L. Huang, "ACO-based hybrid classification system with feature subset selection and model parameters optimization", Neurocomputing, vol. 73, no. 1-3, (2008), pp. 438-448.

[13] K. W. Li and J. Tian, "The multicast routing QoS based on the improved ACO algorithm", Journal of Networks, vol. 4, no. 6, (2009), pp. 505-510.

[14] O. Baskan, S. Haldenbilen, H. Ceylan and H. Ceylan, "A new solution algorithm for improving performance of ant colony optimization", Applied Mathematics and Computation, vol. 221, no. 1, (2009), pp. 75-84.

[15] X. X. Zhang and L. X. Tang, "A new hybrid ant colony optimization algorithm for the vehicle routing problem”, Pattern Recognition Letters, vol. 30, no. 9, (2009), pp. 848-855.

[16] Y. Yi and J. L. Lai, "Computation model and improved ACO algorithm for $\mathrm{p} / / \mathrm{T}$ ”, Journal of Systems Engineering and Electronics, vol. 20, no. 6, (2009), pp. 1336-1343.

[17] B. B. Jiang, H. M. Chen, L. N. Ma and L. Deng, "Time-dependent pheromones and electric-field model: A new ACO algorithm for dynamic traffic routing", International Journal of Modelling, Identification and Control, vol. 12, no. 12, (2011), pp. 29-35.

[18] B. Shuang, J. P. Chen and Z. B. Li, "Study on hybrid PS-ACO algorithm", Applied Intelligence, vol. 34, no. 1, (2011), pp. 64-73.

[19] M. J. Meena, K. R. Chandran, A. Karthik and A. V. Samuel, "A parallel ACO algorithm to select terms to categorize longer documents", International Journal of Computational Science and Engineering, vol. 6, no. 4, (2011), pp. 238-248.

[20] J. Q. Geng, L. P. Weng and S. H. Liu, "An improved ant colony optimization algorithm for nonlinear resource-leveling problems", Computers and Mathematics with Applications, vol. 61, no. 8, (2011), pp. 2300-2305.

[21] L. N. Xing, P. Rohlfshagen, Y. W. Chen and X. Yao, "A hybrid ant colony optimization algorithm for the extended capacitated arc routing problem", IEEE Transactions on Systems, Man, and Cybernetics, Part B: Cybernetics, vol. 41, no. 4, (2011), pp. 1110-1123.

[22] K. Cao, X. Yang, X. J. Chen, Y. L. Zang, J. M. Liang and J. Tian, "A novel ant colony optimization algorithm for large-distorted fingerprint matching", Pattern Recognition, vol. 45, no. 1, (2012), pp. 151161.

[23] Y. C. Li, C. H. G. Ban, S. J. Zhou, X. Q. Li and R. Y. Li "Novel ACO based on entropy and its application in analysis of structural reliability", Journal of Theoretical and Applied Information Technology, vol. 45, no. 1, (2012), pp. 154-159.

[24] N. Zhao, X. W. Lv and Z. L. Wu, "A hybrid ant colony optimization algorithm for optimal multiuser detection in DS-UWB system", Expert Systems with Applications, vol. 39, no. 5, (2012), pp. 5279-5285.

[25] M. J. Meena, K. R. Chandran, A. Karthik and A. V. Samuel, "An enhanced ACO algorithm to select features for text categorization and its parallelization", Expert Systems with Applications, vol. 39, no. 5, (2012), pp. 5861-5871

[26] X. Chen, Y. Y. Kong, X. Fang and Q. D. Wu, "A fast two-stage ACO algorithm for robotic path planning”, Neural Computing and Applications, vol. 22, no. 2, (2013), pp. 313-319.

[27] C. H. Hsu and C. F. Juang, "Evolutionary robot wall-following control using type-2 fuzzy controller with species-DE-activated continuous ACO”, IEEE Transactions on Fuzzy Systems, vol. 21, no. 1, (2013), pp. 100-112.

[28] Y. C. Li, L. P. Liu, S. J. Zhou and Z. C. Yang, "Dynamic resource scheduling in construction project group management based on improved ACO algorithm", Energy Education Science and Technology Part A: Energy Science and Research, vol. 31, no. 2, (2013), pp. 1111-1116.

[29] L. J. Ke, Q. F. Zhang, S. J. Zhou and R. Battiti, "MOEA/D-ACO: A multiobjective evolutionary algorithm using decomposition and AntColony", IEEE Transactions on Cybernetics, vol. 43, no. 6, (2013), pp. 1845-1859.

[30] W. Elloumi, E. A. Haikal, A. Abraham and A. M. Alimi, "A comparative study of the improvement of performance using a PSO modified by ACO applied to TSP”, Applied Soft Computing Journal, vol. 25, (2014), pp. 234-241.

[31] K. Shima and N. P. Hossein, "An advanced ACO algorithm for feature subset selection", Neurocomputing, vol. 147, no. 1, (2015), pp. 271-279.

[32] M. Kefayat, A. L. Ara and S. A. N. Niaki, "A hybrid of ant colony optimization and artificial bee colony algorithm for probabilistic optimal placement and sizing of distributed energy resources", Energy Conversion and Management, vol. 92, (2015), pp. 149-161.

[33] G. M. Xiong, X. Y. Li, S. Zhou, H. Lu, Y. Jing and J. W. Gong, "Incorporating bidirectional heuristic search and improved ACO in route planning", International Journal of Hybrid Information Technology, vol. 8, no. 7, (2015), pp. 189-198. 
[34] J. H. Wu, J. Zhang and Z. H. Liu, "Solving TSP based on adaptive polymorphic immune ant colony algorithm”, Application Research of Computers, vol. 27, no. 5, (2010), pp. 1653-1658.

\section{Authors}

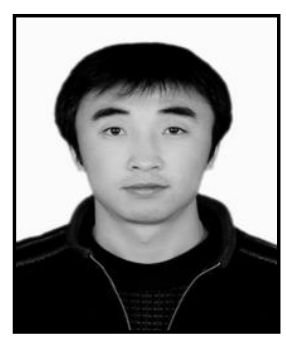

Wenli Lei, Lecture, Ph.D Candidates. He received the M.S degree from Xi an University of Science and Technology in 2008. His research interests include wireless sensor network, artificial intelligence, and so on.

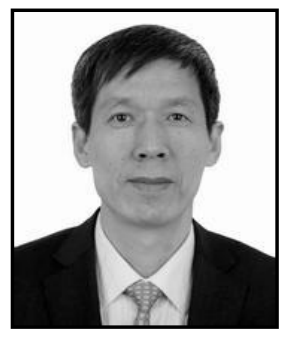

Fubao Wang, Doctor, professor, Ph.D. supervisor. His research interests include computer networks, network and information security, wireless communication networks, digital multimedia communications and so on. 
International Journal of Database Theory and Application Vol.9, No.9 (2016) 